\title{
Estrogen-related receptors: novel potential regulators of osteoarthritis pathogenesis
}

\author{
Jinshuo Tang ${ }^{1}$, Tong Liu' ${ }^{1}$ Xinggui Wen², Zhongsheng Zhou' ${ }^{1}$ Jingtong Yan' ${ }^{1}$ Jianpeng Gao ${ }^{1}$ and Jianlin Zuo ${ }^{1 *}$ (D)
}

\begin{abstract}
Osteoarthritis $(\mathrm{OA})$ is a chronic inflammatory disease that is associated with articular cartilage destruction, subchondral bone alterations, synovitis, and even joint deformity and the loss of joint function. Although current basic research on the pathogenesis of OA has made remarkable progress, our understanding of this disease still needs to be further improved. Recent studies have shown that the estrogen-related receptor (ERR) family members ERRa and ERRY may play significant roles in the pathogenesis of OA. In this review, we refer to the latest research on ERRs and the pathogenesis of OA, elucidate the structure and physiopathological functions of the ERR orphan nuclear receptor family, and systematically examine the relationship between ERRs and OA at the molecular level. Moreover, we also discuss and predict the capacity of ERRs as potential targets in the clinical treatment of OA.
\end{abstract}

Keywords: Estrogen-related receptors, ERRs, Pathogenesis, Osteoarthritis

\section{Introduction}

Osteoarthritis (OA) is the most common joint disease among the elderly population (Glyn-Jones et al. 2015). Approximately one-third of senior citizens over 65 years of age suffer from $\mathrm{OA}$, and the incidence is significantly higher in women than in men. According to epidemiological survey data (Johnson and Hunter 2014), with the aging of the population and the increase in average life expectancy, the incidence and prevalence of OA are soaring. However, the current treatment provided by clinicians for OA patients is still limited to symptom management (Correa and Lietman 2017; DeRogatis et al. 2019), which fails to curb the development of this condition. We now realize that inflammatory cytokines, metalloproteinases, cellular senescence, estrogen and biomechanical imbalances play crucial roles in the progression of $\mathrm{OA}$ and can lead to a series of critical pathologic changes (Wang et al. 2017a; Mehana et al. 2019; McCulloch et al. 2017; Watt 2016), such as focal cartilage

\footnotetext{
*Correspondence: zuojl@jlu.edu.cn

${ }^{1}$ Department of Orthopeadics, China-Japan Union Hospital of Jilin University, Changchun 130033, Jilin, China

Full list of author information is available at the end of the article
}

deficiency, osteophyte formation, subchondral bone remodeling and synovial hyperplasia, in the joints of $\mathrm{OA}$ patients (Charlier et al. 2019), but our understanding of the pathogenesis of OA still needs to be improved. In recent years, studies have shown that estrogen-related receptor $\alpha(E R R \alpha)$ and $\gamma(E R R \gamma)$ in the estrogen-related receptor (ERR) family may play essential roles in the pathogenesis of OA. In this review, we systematically expounded on the relationship between ERRs and OA at the molecular level by referring to recent research findings.

\section{Structure of estrogen-related receptors}

ERRs are members of the nuclear receptor superfamily and have a tight structural relationship with estrogen receptor $\alpha(E R \alpha)$ and $\beta(E R \beta)$ (Eichner and Giguere 2011). In 1988, two unique nuclear receptors with conserved steroid hormone receptor features were identified by a probe synthesized from a cDNA library, namely, estrogen-related receptors $\alpha$ and $\beta$ (Giguère et al. 1988). Subsequently, in 1998, the third receptor isoform, estrogen-related receptor $\gamma$, was discovered by researchers (Eudy et al. 1998). The ERR family have extensive sequence similarity with the DNA-binding domain 
(DBD) and ligand-binding domain (LBD) of ER $\alpha$ (Divekar et al. 2016). However, these receptors cannot bind to endogenous estrogen or its derivatives, and so ERRs are also referred to as orphan nuclear receptors (Huss et al. 2015; Tripathi et al. 2020). In human tissue, ERR $\alpha$ has no known splice variants, ERR $\beta$ has three splice variants, and ERRY has two splice variants (Xu et al. 2016). These splice variants signify a significant source of functional diversity in the proteome (Heckler and Riggins 2015); for instance, the expression of the ERR $\beta L$ splice variant can augment ER $\alpha$-dependent gene activation (Bombail et al. 2010), and activated ERR $\beta 2$ splice variants are potent inhibitors of karyokinesis in breast carcinoma cells, including TNBC (Heckler et al. 2016). However, there are still quite a few limitations in the understanding of these splice variants due to the limited research currently available (Bombail et al. 2010; Bielli et al. 2019).

The molecular structure of ERRs is similar to that of other nuclear receptors, and these proteins consist of six conserved regions (A/B, C, D, E/F domains) (Lu et al. 2019) (Fig. 1). The N-terminal region is the $\mathrm{A} / \mathrm{B}$ domain, also known as activation domain-1 (AF-1), and has the characteristics of ligand-independent transcriptional activation. The A/B domains of ERRs contain conserved motifs that allow their transcriptional activity to be regulated by posttranslational modifications such as phosphorylation and SUMOylation (Vu et al. 2007; Tremblay et al. 2008).

The central C domain of ERRs is referred to as the DBD and contains two highly conserved zinc finger motifs (Saito and Cui 2018), which can bind to a specific DNA sequence (TCAAGGTCA) called the ERR response element (Xia et al. 2019). ERRs can bind with ERR monomers, homodimers or heterodimers consisting of two different ERR isoforms (Mohideen-Abdul et al. 2017; Horard et al. 2004). Because all members of the ERR family have virtually identical $C$ domains, distinct isoforms of ERR can sometimes target the same gene (Casaburi et al. 2018).

The $\mathrm{D}$ domain is a flexible hinge region that provides protein flexibility when the dimer is bound to DNA and links the $\mathrm{C}$ and $\mathrm{E}$ regions (Misra et al. 2017; Helsen and Claessens 2014).

The E/F domain is the ligand-binding domain. The LBDs of ERRs and ER $\alpha$ share 30-40\% homology. However, ERRs cannot bind to endogenous estrogen or its derivatives because they lack Cys residues that identify ligands (Gibson and Saunders 2012). The LBD contains a

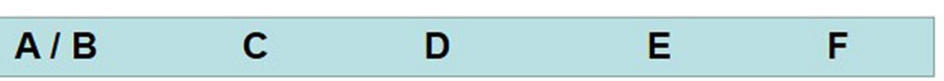

A. ERR $\alpha$ and $E R \alpha$

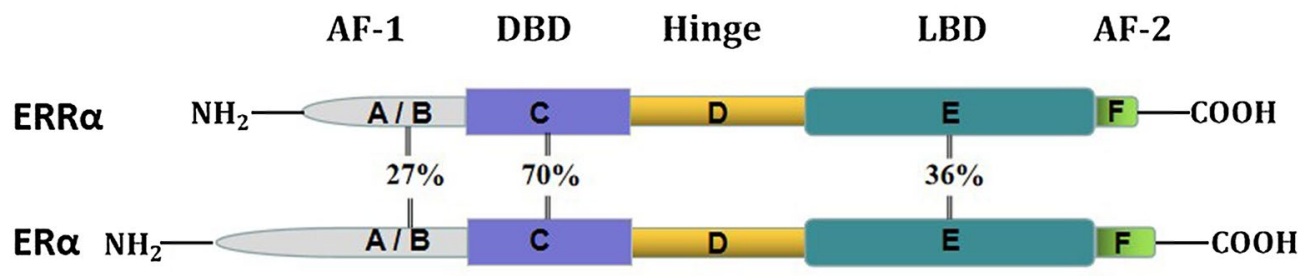

B. Three ERR isoforms

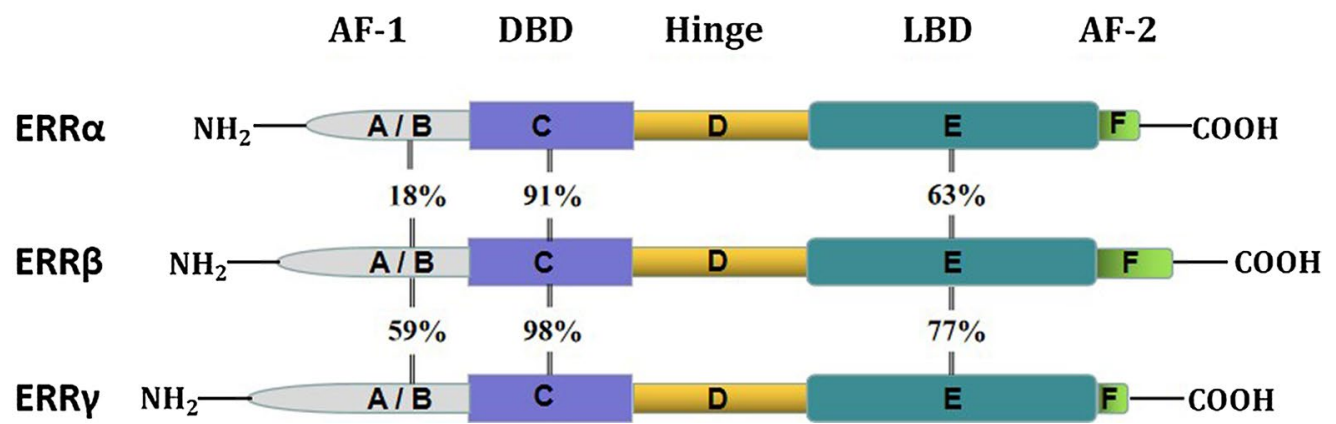

Fig. 1 Structure of estrogen receptor $a$ and the three estrogen-related receptors. ERRs are composed of six conserved regions (A/B, C, D, and E/F domains), and the colors symbolize the diverse functions of these domains. The number between the two receptors represents the sequence identity of the same domain in the different receptors 
conserved helix motif called activation function-2 (AF-2) (Huss et al. 2015), and this motif is exposed. Three ERRs are constitutively active due to the structure of the E/F domain, which is capable of binding coregulators in the absence of ligand binding (Misawa and Inoue 2015; Liu et al. 2016; Chen et al. 2001). Although the ligand binding pockets of ERRs are smaller than those of ER $\alpha$, it is difficult to accommodate effective natural ligands (Greschik et al. 2002). However, it is hypothesized that the transcriptional activity of ERRs can still be regulated by certain undiscovered ligands (Gibson and Saunders 2012). A large number of experiments have shown that the use of some synthetic compounds can regulate the function of ERRs. For example, compound LingH2-10 is a novel selective inverse agonist of ERR $\alpha$ (Ning et al. 2017), compound DY181 is also considered to be a selective inverse agonist of ERR $\beta$ and has excellent selectivity and effectiveness (Yu et al. 2017), and compound DY40 is a synthetic inverse agonist of ERR $\gamma$.

Protein sequence analysis of all members of the ERR family revealed that the DBDs and LBDs of the three ERR isoform have high amino acid sequence homology, suggesting that they may bind to similar ligands and target the same promoter and enhancer elements (Giguère 2002). The ERR family has the highest DBD amino acid sequence identity (91-98\%), relatively low sequence homology among LBDs (62-77\%), and lowest sequence identity for $\mathrm{A} / \mathrm{B}$ domains compared to DBDs and LBDs (15-59\%).

\section{Physiological and pathological function of ERRs}

The physiological functions of ERRs are complex and varied, and these proteins play crucial roles in controlling the balance of cellular metabolism, general metabolism, growth and development, cancer occurrence, and bone homeostasis (Villena and Kralli 2008; Thouennon et al. 2019; Misra et al. 2016; Zhang et al. 2015; Li et al. 2019a). The expression of ERRs is widespread and is particularly high in tissues with high energy expenditure or vigorous metabolic demands (Festuccia et al. 2018). Among adults, Esrra exhibits the highest expression level, Esrrg exhibits an intermediate level of expression, and Esrrb shows the lowest expression (Likhite et al. 2019). As the site of oxidative metabolism, the metabolic activity of mitochondria is strictly controlled to meet the energy demands of cells under different physiological conditions. The wellknown inducers of mitochondrial oxidative metabolism are peroxisome proliferator-activated receptor $\gamma$ coactivator $1 \alpha(\mathrm{PGC} 1 \alpha)$ and nuclear receptor corepressor 1 (NCOR1) (Brown et al. 2018b; Lima et al. 2018), which are abundantly expressed in high-energy demand tissues such as the heart, skeletal muscle, and brown adipose tissue (BAT). However, both PGC1 $\alpha$ and NCOR1 lack DNA binding activity and depend on interactions with transcription factors that directly bind and control downstream target genes. ERRs have been shown to be key transcription factors that regulate mitochondrial oxidative metabolism and induce PGC1 $\alpha$ and NCOR1 expression (Fan and Evans 2015). Studies have shown that ERRs can bind and regulate the expression of glycolytic genes (Long et al. 2020), including pivotal enzymes such as phosphofructokinase, hexokinase 2 (HK2), glyceraldehyde phosphate dehydrogenase (GAPDH) and enolase 1 (ENO1), which are crucial components of cellular glucose metabolism (Kida et al. 2015). ERR $\alpha$ is highly expressed in tissues involved in lipid metabolism and energy balance, such as white adipose tissue (WAT) and BAT, the heart and skeletal muscles, which require high oxidative capacity (Audet-Walsh and Giguere 2015). Mice lacking adipose ERRs (ERR $\alpha \gamma \mathrm{Ad}-/-$ ) have reduced oxidative and thermogenic capacity, and when exposed to a low-temperature environment, they rapidly become hypothermic (Brown et al. 2018a). The thermogenesis of BAT depends on the level and activity of mitochondrial uncoupling protein 1 (UCP1) (Oelkrug et al. 2015). Epinephrine stimulates BAT cells to activate UCP1-mediated thermogenesis (Porter 2017), which also stimulates UCP1 gene expression. Recent studies have shown that ERR isoforms function in BAT in a highly complementary manner to control mitochondrial biogenesis and cellular oxidative capacity. There are defects in the transcription and metabolic reaction of BAT lacking all ERRs to adrenaline-stimulated UCP1 (Gantner et al. 2016), resulting in a significant decrease in mitochondrial content and oxidative capacity. ERRs are vital effectors of adrenalinestimulated BAT transcriptional reprogramming.

The high expression of ERR $\alpha$ is related to the poor prognosis of numerous malignancies and can promote the invasive characteristics of a variety of cancers (Tribollet et al. 2016). In estrogen receptor-negative (ER-) breast cancer cells, ERR $\alpha$ acts as an activating transcription factor. ERR $\alpha$ overexpression increases the growth of breast cancer cells in the mammary gland, as well as the expression of vascular endothelial growth factor (VEGF) (Misawa and Inoue 2015; Fradet et al. 2011). In triplenegative breast cancer (TNBC), ERR $\alpha$ can also regulate the expression of genes needed for cancer cell metabolism, enhancing the ability of breast cancer cells to use lactic acid as a metabolic substrate (Park et al. 2016). Furthermore, ERR $\alpha$ enhances breast cancer resistance to certain anticarcinogens by regulating mitochondrial metabolic adaptation ( $\mathrm{Li}$ et al. 2020). The combined administration of an ERR $\alpha$ inhibitor and rapamycin to ER - breast carcinoma cells can synergistically suppress the proliferation of tumor cells (Berman et al. 2017). In contrast, ERR $\alpha$ expression is upregulated in urinary 
bladder carcinoma. After inhibiting the expression of ERR $\alpha$, the growth, proliferation, invasion and migration of bladder carcinoma cells are inhibited, promoting cancer cell apoptosis and inhibiting the epithelial-mesenchymal transition (EMT) of tumor cells (Ye et al. 2019). ERRs can directly and/or indirectly affect the physiological and molecular characteristics of tumor Leydig cells via the formation of a microenvironment (Kotula-Balak et al. 2018). ERR $\alpha$ in endometrial carcinoma cells plays a critical role in TGF- $\beta$-induced EMT through cancerstromal interactions (Yoriki et al. 2019).

Interestingly, ERRs have also beneficial effects on disease treatment in the context of tumorigenesis and development. ERR $\alpha$ can also serve as an activating transcription factor or a transcriptional repressor depending on the cellular microenvironment, thereby promoting or inhibiting tumor growth in breast cancer (Misawa and Inoue 2015). In some patients with TNBC, the high expression of ERR $\alpha$ is a biomarker of the patients' response to tamoxifen and a favorable prognostic factor for tamoxifen treatment (Manna et al. 2016). The overexpression of ERR $\beta$ or ERR $\gamma$ can inhibit the proliferation of prostate cancer cells, and some research findings indicate that the expression of ERR $\beta$ or ERR $\gamma$ in prostate carcinoma is frequently diminished (Misawa and Inoue 2015). The ERR $\gamma$ agonist DY131 suppresses cancer growth and inhibits the Wnt signaling pathway. ERRY is a novel tumor inhibitor that can block Wnt signaling and is a potential therapeutic target for gastric carcinoma (Kang et al. 2018).

ERRs have significant physiological and pathological effects on bone tissue. ERR $\alpha$ plays a role in tumor bone metastasis, which can occur in up to $70 \%$ of patients with advanced breast cancer, and ERR $\alpha$ can play multiple roles to promote the invasion of bone tissue by primary tumors (Misawa and Inoue 2015). In bones, the effects of cholesterol, statins, and bisphosphonates on osteoclast formation require ERR $\alpha$. Both cholesterol-induced bone loss and bisphosphonate-mediated protective effects are lost in an ERR $\alpha$-knockout (KO) mouse model (Wei et al. 2016). ERR $\alpha$ regulates bone remodeling by controlling osteoclastogenesis, which is a necessary cell differentiation process for bone resorption. The deletion of ERR $\alpha$ disrupts osteoclast differentiation and inhibits bone resorption (Wan 2010).

Additionally, ERR $\alpha$ inhibits osteoblastic differentiation (Gallet and Vanacker 2010). Another study showed that ERR $\alpha-K O$ mice were resistant to bone loss, and compared with those of wild-type mice, the number and activity of osteoclasts remained unchanged, while the bone formation rate and the activity of osteoblasts increased (Zhang et al. 2016). Some studies mainly point to ERR $\alpha$ as a switch that represses the differentiation of precursor cells into the osteoblastic pathway while favoring the adipocytic pathway (Gallet and Vanacker 2010). ERR $\alpha$ deficient mice exhibited mild increases in cancellous bone volume and the amount of bone surfaces covered with bone-forming osteoblasts, whereas bone marrow fat volume was decreased (Delhon et al. 2009).

Moreover, ERR $\gamma$ negatively regulates osteoblast differentiation and bone formation (Jeong et al. 2009). Bone trabeculae in $E R R \gamma^{+/-}$heterozygous mice lacking the $E R R \gamma$ gene were increased compared to those of control animals (Cardelli and Aubin 2014). However, ERRY is strongly expressed in bone marrow-derived macrophages (BMMs), which are osteoblast precursors; ERR $\gamma$ suppresses the formation of multinucleated osteoclasts and attenuates the induction of nuclear factor of activated $\mathrm{T}$ cells $\mathrm{c} 1$, which is a critical modulator of osteoclastogenesis (Kim et al. 2019a). Suppressing ERR $\alpha$ and/or ERR $\gamma$ can boost bone formation and compensate for bone loss due to aging or estrogen deficiency (Carnesecchi and Vanacker 2016). These experiments have fully demonstrated that ERR $\alpha$ and ERR $\gamma$ play significant roles in maintaining bone homeostasis.

\section{Functions of ERRs in the pathogenesis of osteoarthritis}

ERRs are highly expressed in the bone and cartilage tissue of the extremities and trunk, and they play essential roles in maintaining tissue homeostasis (Bonnelye et al. 1997; Bonnelye and Aubin 2005; Lorenzo 2017). Previous studies have shown that the ERRs, which are dominated by ERR $\alpha$ and ERR $\gamma$, are significantly associated with OA (Fig. 2).

\section{Estrogen-related receptor $a$}

$E R R \alpha$ has dual functions in the occurrence and development of OA. On the one hand ERR $\alpha$ may response to the healing signal promotes the cartilage formation by upregulating Sry-type high-mobility-group box transcription factor 9 (Sox-9), but on the other, it can accelerate progression of OA in multiple ways (Bonnelye et al. 2011).

The effect of ERR $\alpha$ on cartilage formation is mainly associated with the regulation of its target gene of Sox-9 (Chen et al. 2014), which is involved in the proliferation, differentiation and maturation of chondrocytes. Sox-9 is a key regulator of chondrogenic differentiation and cartilage formation (Liu et al. 2018; Wu et al. 2018). ERR $\alpha$ directly or indirectly upregulates Sox-9 gene expression in chondrocytes (Bonnelye et al. 2007), thereby promoting the proliferation and accumulation of cartilage precursor cells and further induces the differentiation of these cells into mature chondrocytes; these cells participate in gristle formation, which plays an important role in maintaining the integrity of cartilaginous tissue (Bonnelye and Aubin 2005). The effect of ERR $\alpha$ on cartilage 


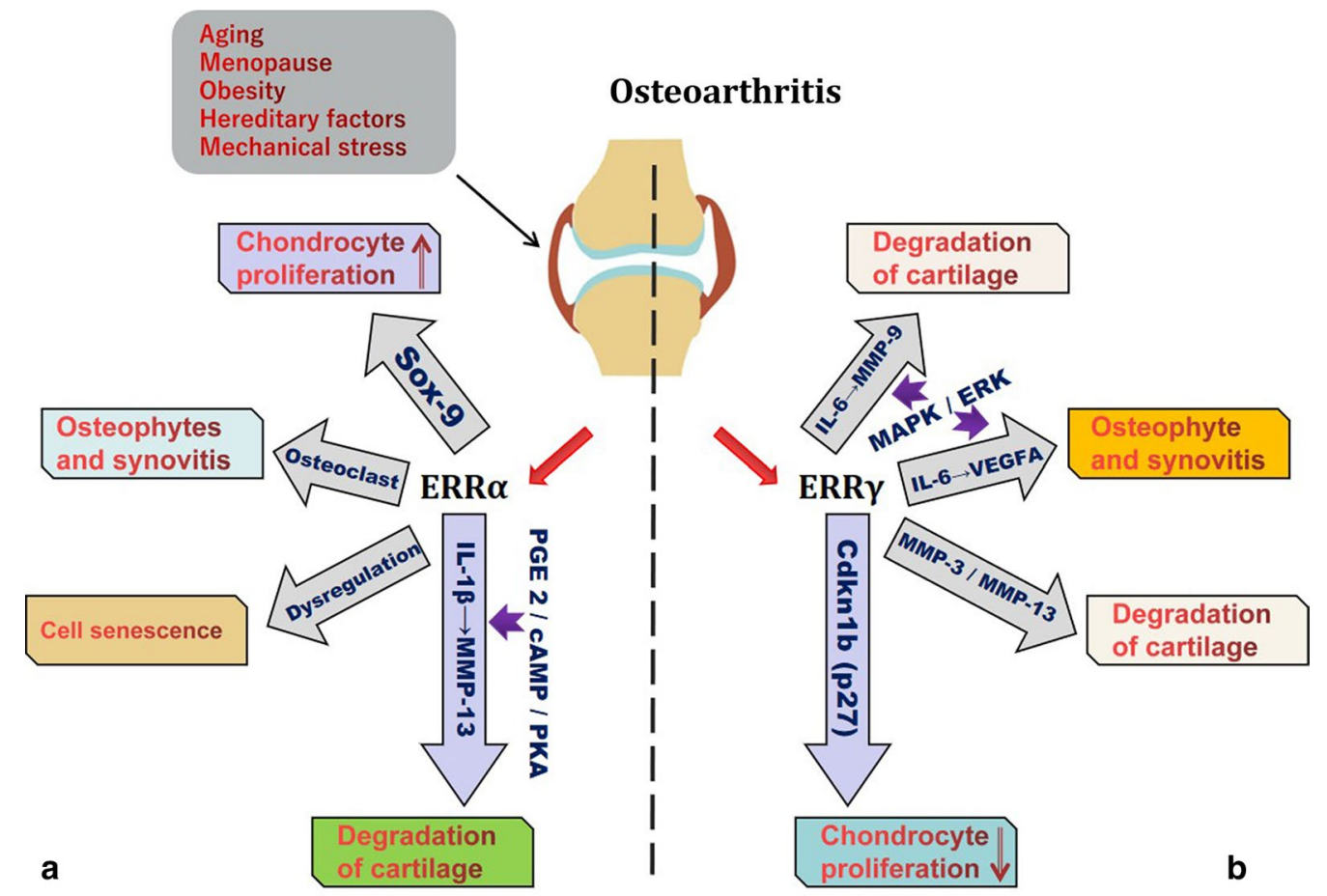

Fig. 2 When the joint is affected by pathogenic factors such as aging, menopause, obesity, hereditary factors, and mechanical stress, the expression of the ERR family becomes disordered. a ERRa promotes chondrocyte proliferation by upregulating Sox-9. ERRa is generated in response to IL-1 $\beta$ stimulation through the PGE2/CAMP/PKA signaling pathways and regulates MMP-13 production. The dysregulation of ERRa may affect the aging of chondrocytes. ERRa participates in osteophyte formation and synovial hyperplasia by impacting osteoclasts. $\mathbf{b}$ The overexpression of the ERRY, as regulators of IL-6, VEGFA and MMPs, will inevitably result in the dysfunction of molecular substances, disrupt homeostasis, and induce osteophyte, synovitis and cartilage degradation. ERRY also restrains chondrocyte proliferation by upregulating p27

in vertebrate embryo development was verified in a zebrafish embryo model. In the zebrafish embryo model with ERR $\alpha$ gene knockout, the expression of Sox-9 was significantly lower than that in the equivalent wild-type embryo model during growth and development, which leads to malformation of the pharyngeal arch cartilage of the embryo in the course of upgrowth (Kim et al. 2015). Bonnelye et al. collected femoral condyles and tibial plateaus from OA patients after total knee arthroplasty to isolate and culture OA chondrocytes in vitro. OA chondrocytes were treated with the XCT790, a synthetic reverse agonist of ERR $\alpha$, for $24 \mathrm{~h}$, and the expression index of Sox-9 in OA chondrocytes was dosedependently downregulated by XCT790 (Kokabu et al. 2019). This experiment indirectly confirmed that ERR $\alpha$ slows OA chondrocyte loss by participating in cartilage formation.

The ERR $\alpha$-mediated degradation of cartilage is associated with interleukin-1 $\beta$ (IL-1 $\beta$ ) and matrix metalloproteinase-13 (MMP-13). IL-1 $\beta$ is an inflammatory cytokine that is closely related to the occurrence and development of OA (Liao et al. 2020; Wang and He 2018) and can participate in the pathological changes in OA through many mechanisms. When human OA chondrocytes were treated with IL- $1 \beta$ for $24 \mathrm{~h}$, the expression of ERR $\alpha$ increased (Bonnelye et al. 2011). Further research showed that IL-1 $\beta$ stimulated ERR $\alpha$ expression via the PGE2/cAMP/PKA signaling pathway. MMP-13, also known as collagenase 3 , performs a significant role in the process of OA (Li et al. 2017; Chan et al. 2017). MMP13 can induce gristle damage by degrading collagens and proteoglycans in the cartilaginous extracellular matrix (ECM) (Fosang et al. 1996; Zhang et al. 2018a), and IL- $1 \beta$ is one of the major cytokines that induces MMP13 expression (Tabeian et al. 2019). ERR $\alpha$ is generated in response to IL- $1 \beta$ stimulation through the PGE2/cAMP/ PKA signaling pathways and is an important orphan nuclear receptor that regulates MMP-13 production. The level of IL-1-induced MMP-13 mRNA in OA chondrocytes was dose-dependently decreased by XCT790 (Bonnelye et al. 2011), which demonstrated that ERR $\alpha$ could upregulate the expression of MMP-13. ERR $\alpha$ is involved in IL- $1 \beta$-mediated OA cartilage degradation and accelerates the progression of cartilage loss.

Osteocyte and chondrocyte senescence is one of the vital causes leading to the initiation and development 
of OA (Rahmati et al. 2017; Millerand et al. 2019). In chondrocytes in human OA, the expression of ERR $\alpha$ is dysregulated, and this condition becomes more common with age (Bonnelye and Aubin 2013), which indicates that ERR $\alpha$ may be a significant regulator of cellular senescence, thereby affecting the aging of chondrocytes (Huang et al. 2017).

ERR $\alpha$ is a crucial conditioning agent that promotes osteoclastogenesis and oxidative metabolism and is involved in many processes (Bae et al. 2017; Yang and Wan 2019), such as cell adhesion and transport, when expressed in osteoclasts (Bonnelye et al. 2010). ERR $\alpha$ is also expressed in highly motile cells such as macrophages and is an important regulator of biological functions (Leopold Wager et al. 2019). Macrophages lacking ERR $\alpha$ gene expression have decreased cell viability due to decreases in intracellular mitochondrial gene expression and reactive oxygen species (ROS) levels (Sonoda et al. 2007). A comprehensive analysis of the relationship between ERR $\alpha$, osteoclasts and macrophages supports the role of ERR $\alpha$ in inflammatory diseases, such as OA and rheumatoid arthritis, that are associated with osteoclast-induced bone degradation leading to bone degeneration (Bonnelye et al. 2010). For example, enhanced subchondral bone remodeling and synovitis are the main pathological manifestations of osteoarthritis (Aho et al. 2017; Mathiessen and Conaghan 2017). The former is primarily characterized by macrophage infiltration and osteoclastogenesis (Zhu et al. 2019), while the latter is chiefly characterized by the infiltration of inflammatory cells such as macrophages and vascular proliferation (Zhang et al. 2020). Researchers verified the role of ERR $\alpha$ in osteoclasts in ERR $\alpha-K O$ mice and showed that ERR $\alpha$ deletion disrupted the expression of several major genes in cells, and ERR $\alpha-K O$ mice exhibited osteopetrosis due to osteoclast defects and decreased bone resorption, suggesting that ERR $\alpha$ may be a significant regulator of osteoclastogenesis (Yang and Wan 2019). However, while $E R R \alpha$ may participate in the occurrence and development of OA by regulating osteoclasts and macrophages, sufficient experimental evidence is required to elucidate its specific mechanism of action.

\section{Estrogen-related receptor $\gamma$}

ERR $\gamma$ can upregulate matrix metalloproteinase-9 (MMP9) expression via the IL-6-mediated MAPK/ERK pathway and thus has an essential role in the destruction of OA cartilage (Son et al. 2017). An OA mouse model was used to show that the expression of ERRY in cartilage was significantly higher than that in wild-type mouse cartilage. When ERRy expression was inhibited by ERR siRNA or GSK5182, a reverse agonist of ERR (Kim et al. 2016), to inhibit its transcriptional activity, the expression of
MMP-9 decreased when chondrocytes were stimulated with IL-6 (Son et al. 2017). It has been demonstrated that in OA chondrocytes, IL-6 mediates ECM degradation by stimulating the expression of MMP-9, and the overexpression of ERR $\gamma$ amplifies this effect.

ERRY can participate in the occurrence and development of OA through vascular endothelial growth factor A1 (VEGFA). IL-6 can stimulate chondrocytes to produce angiogenic factors, such as VEGFA (Son et al. 2017). The expression of VEGFA in OA is significantly related to the severity and pain intensity in OA (Hamilton et al. 2016; Guan et al. 2020), which is also crucial for osteophyte development (Wang et al. 2017b). After osteophytes are formed, the continuous production of VEGFA further stimulates vascular proliferation by osteophytes (Hashimoto et al. 2002). Another function of VEGFA is to stimulate the proliferation of OA synovium and synovial blood vessels, leading to inflammatory cell infiltration of the synovium and pain in patients with OA (Semerano et al. 2016). IL-6 is an important cytokine that regulates VEGFA gene expression (Kayakabe et al. 2012). $E R R \gamma$ can regulate VEGFA gene expression by participating in the MAPK/ERK signaling pathway downstream of IL-6. Animal experiments indicated that the expression level of VEGFA in OA chondrocytes was reduced after using ERR siRNA to inhibit ERR $\gamma$ expression or GSK5182 to inhibit ERR $\gamma$ transcription (Son et al. 2017). ERR affects the expression of MMP-9 and VEGFA through the MAPK/ERK signaling pathway in chondrocytes, thereby participating in pathological processes such as cartilage degradation, vascular proliferation, osteophyte formation and synovial hyperplasia in the course of OA.

Experimental mouse models of OA were established by destabilization of the medial meniscus (DMM) surgery (Hong et al. 2020). Eight weeks later, the expression of ERRY in the cartilage of OA mice was significantly increased compared with that in wild-type mice (Zhao et al. 2019), while the expression of matrix metalloproteinase-3 (MMP-3) and MMP-13 increased, and DMM mice showed significant $O A$ manifestations, including cartilage erosion, subchondral osteosclerosis and osteophyte formation (Fang et al. 2018; Tetlow et al. 2001). When the experimental mice were replaced with Esrrg $^{+/-}$heterozygous mice lacking one Esrrg allele, the OA mouse model was then established by DMM surgery. Eight weeks later, the researchers examined heterozygous mice for the lack of an allelomorph of Esrrg, and the expression level of ERRY in joint tissue was reduced (Son and Chun 2018), the expression levels of MMP-3 and MMP-13 induced by DMM were dramatically reduced, and the OA symptoms of cartilage erosion, subchondral osteosclerosis and osteophyte formation were significantly decreased compared with those of normal DMM 
mice (Zhao et al. 2019). This experiment shows that ERR $\gamma$ upregulates the levels of MMP-3 and MMP-13 in articular chondrocytes via overexpression (Son and Chun 2018), which in turn leads to the degradation of collagens and proteoglycans in cartilage ECM and the exacerbation of OA symptoms (Guo et al. 2017; Hardy and FernandezPatron 2020). ERRY is a novel regulator of the pathogenesis of osteoarthritis.

Transgenic technology was used to generate ERR $\gamma$ overexpressing mouse models, and the expression level of ERR $\gamma$ in bone and cartilage was significantly higher than that in wild-type mice. Quantitative analysis of the proximal humerus, distal femur and proximal tibia revealed that the cartilage growth plate was obviously smaller than that of wild-type mice (Cardelli et al. 2013), and the declining regions were principally concentrated in the zone of proliferating cartilage (Michigami 2013). Compared with that of the control group, the height of the proliferating cartilage zone in the experimental group was decreased by $22 \%$, and the primary reason for this dramatic decrease was that chondrocyte proliferation, differentiation, maturation and other behaviors were all affected (Cardelli et al. 2013; Hirota et al. 2018). Further studies demonstrated that the main trigger for the inhibition of chondrocyte proliferation ERR $\gamma$-mediated inhibition of chondrocyte proliferation by upregulating the expression of cyclin-dependent kinase inhibitor 1B (p27) (Kashiwagi et al. 2010), thus affecting the formation of cartilage growth plates. These studies show that ERR $\gamma$ is a negative regulator of chondrocyte proliferation and differentiation, and its function appears to be opposite to that of ERR $\alpha$ (Bonnelye et al. 2011). In the occurrence and development of OA, the proliferation and differentiation of chondrocytes can slow the damage to articular cartilage (Charlier et al. 2019; Harrell et al. 2019). In summary, however, the expression of ERR $\gamma$ in the cartilage of OA mice was significantly higher than that of wild-type mice (Zhao et al. 2019). Overexpression of ERR $\gamma$ may affect the proliferation and differentiation of chondrocytes, which is detrimental to the process of self-repair in OA cartilage.

Bone and cartilage tissue homeostasis is coregulated by a variety of cytokines, growth factors and metalloproteases, and numerous molecular substances can synergetically or antagonistically maintain homeostasis (Mehana et al. 2019; Wojdasiewicz et al. 2014; Boehme and Rolauffs 2018). When bone and cartilage tissues are affected by pathogenic factors such as age, menopause, obesity, heredity, and mechanical stress, the expression of the ERR family, as regulators of IL-1/IL-6, Sox-9 and other molecular substances, becomes disordered (Loeser et al. 2012). Dysregulated expression will inevitably result in the dysfunction of cytokines, growth factors and metalloproteinases, disrupt homeostasis, and induce articular cartilage ECM degradation, synovial hyperplasia, osteophyte formation and other pathological manifestations in the occurrence and development of OA, as well as affect the duration of symptoms.

\section{ERRs and the development of innovative drugs}

Because we generally lack a systematic and specific understanding of the pathogenesis of $\mathrm{OA}$, the treatment options for early-stage patients are still limited (Dadabo et al. 2019; Murphy et al. 2016). Current OA treatment measures are symptomatic treatments but not etiological treatments. At best, these treatments can only alleviate pain and frequently fail to effectively curb the development of OA (Vinatier et al. 2016). Although joint replacement can ameliorate the condition of patients with advanced OA (Gademan et al. 2016), it is expensive, and artificial prostheses have limited service lives. In the case of postoperative complications such as prosthesis loosening and periprosthetic infection, revision surgery is required, which brings enormous psychological pressure and financial burden to patients (Kulshrestha et al. 2019; Schwartz et al. 2020). Based on the role of ERRs in the pathogenesis of osteoarthritis, the use of ERR molecular modulators to treat OA has a certain theoretical basis (Table 1) and important biomedical significance for the development of optimal therapies for the prevention and treatment of OA. Studies have shown that certain ERRrelated inhibitors have good therapeutic effects on ERRmediated diseases (Tripathi et al. 2020). For example, one ERR $\gamma$ reverse agonist is a tetrasubstituted olefin analog that enhances the function of sodium iodide transporters in anaplastic thyroid cancer cells, thereby promoting the response to radioactive iodine treatment in vitro, and can be used as a potential therapeutic agent for ERR $\gamma$ mediated cancers (Kim et al. 2019b). Moreover, diethylstilbestrol (DES) can be used as a reverse agonist for all three ERR isoforms (Gibson and Saunders 2012; Greschik et al. 2002). Compound LingH2-10 is a novel selective inverse agonist of ERR $\alpha$ and can inhibit the growth of TNBC cells (Ning et al. 2017). Compound GSK5182 is an ERRY inverse agonist (Zhang et al. 2018b; Kim et al. 2013) that can strengthen the antitumor efficacy of the tumorreducing drug paclitaxel (Vernier et al. 2020). The compounds GSK 4716 and DY 131 are synthetic ERR $\beta / E R R \gamma$ agonists. These synthetic compounds have conspicuous inverse or positive agonistic effects on ERRs, and they may be used as effective drugs for the prevention and treatment of $\mathrm{OA}$ in the future. However, there are quite a few types of ERR inhibitors, their chemical structures and biological functions are exceedingly complex, and our understanding of their mechanisms of action is still far from complete. Because long-term use of DES increases 
Table 1 Some small molecular modulators of estrogen-related receptors

\begin{tabular}{|c|c|c|c|c|}
\hline Physiological effects & Molecular modulators & Targets & Notes & References \\
\hline \multirow[t]{11}{*}{ Inverse agonists } & ХСТ790 & ERRa & $\begin{array}{l}\text { The detailed molecular mechanism } \\
\text { of XCT790 binding to ERRa remains } \\
\text { ambiguous }\end{array}$ & Kokabu et al. (2019), Vitto et al. (2019) \\
\hline & Ling $\mathrm{H} 2-10$ & ERRa & $\mathrm{IC}_{50}=0.64 \pm 0.12 \mu \mathrm{M}$ & Ning et al. (2017) \\
\hline & Thiazolidinediones & ERRa & & Patch et al. (2011) \\
\hline & Statins & ERRa & $\begin{array}{l}\text { Inhibiting effect in vivo; Inverse effect } \\
\text { in vitro }\end{array}$ & Tripathi et al. (2020) \\
\hline & DY40 & ERR $\beta$ & $\begin{array}{l}\text { The most potent ERR } \beta \text { inverse } \\
\text { agonist }\end{array}$ & Yu et al. (2017) \\
\hline & GSK5182 & ERRY & $\begin{array}{l}\text { Relatively non-toxic with an oral } \mathrm{LD}_{50} \\
\text { in mice of greater than } 1000 \mathrm{mg} / \mathrm{kg}\end{array}$ & Joo et al. (2015) \\
\hline & DY181 & ERRY & $\begin{array}{l}\text { The most potent ERRy inverse ago- } \\
\text { nist; } I C_{50}=0.01 \mu \mathrm{M}\end{array}$ & Yu et al. (2017) \\
\hline & Tetrasubstituted olefin analog & ERRY & & Kim et al. (2019b) \\
\hline & $4-\mathrm{OHT}$ & ERR $\beta$, ERRY & & Coward et al. (2001) \\
\hline & TAM & ERRß, ERRY & & Coward et al. (2001) \\
\hline & DES & ERRa, ERR $\beta$, ERRY & $\begin{array}{l}\text { Long-term use of DES can increase } \\
\text { the risk of malignant tumors of the } \\
\text { genital system }\end{array}$ & Lu et al. (2012) \\
\hline \multirow[t]{5}{*}{ Agonists } & Cholesterol & ERRa & $\begin{array}{l}\text { The detailed molecular mechanism } \\
\text { of cholesterol binding with ERRa } \\
\text { remains ambiguous }\end{array}$ & Casaburi et al. (2018); Li et al. (2019b) \\
\hline & DY 131 & ERR $\beta$ & & Tiek et al. (2019) \\
\hline & GSK4716 & ERRY & $\begin{array}{l}\text { A potent ERRY agonist with excellent } \\
\text { selectivity over ERRa and ERRß }\end{array}$ & Kim et al. (2009) \\
\hline & Flavone and isoflavone & ERRa, ERR $\beta$ & & Suetsugi et al. (2003) \\
\hline & GSK9089 & ERRß, ERR $\gamma$ & & Zuercher et al. (2005) \\
\hline
\end{tabular}

the risk of malignant tumors in the reproductive system, this drug was restricted in 1971 (Huo et al. 2017; Titus et al. 2019; Smith et al. 2012). The compound XCT790 has always been regarded as a specific inverse agonist of ERR $\alpha$, and it has been widely used in experiments related to ERR $\alpha$ (Kokabu et al. 2019). It is believed that XCT790 has the ability to disrupt the interaction between ERR $\alpha$ and PGC- $1 \alpha$ and inhibit the growth of breast cancer cells. However, some studies suggest that XCT790 does not appear to be a very specific ERR $\alpha$ inverse agonist because at nanomolar concentrations, which is tenfold lower than the concentration required to inhibit ERR $\alpha, \mathrm{XCT} 790$ is an effective, fast-acting mitochondrial uncoupler that enables rapid ATP depletion, and its effect is independent of ERR $\alpha$ inhibition (Vitto et al. 2019). Therefore, further research is needed to elucidate the mechanisms of action of ERR-related inhibitors on joints and other tissues to find innovative drugs to prevent and treat OA.

\section{Conclusion}

ERR $\alpha$ and ERR $\gamma$, which are typical orphan nuclear receptors, can regulate inflammatory cytokines and growth factors and thus exert significant effects on the occurrence and development of OA. Through the progress of basic experimental research, the roles of ERRs in OA have become clearer, while their mechanisms of action still require further study. More research on ERRs in osteoarthritis will provide an additional scientific basis for thoroughly understanding the pathogenesis of OA. The ultimate purpose is to identify drugs to prevent and treat movement system diseases such as OA based on the regulatory actions of ERRs. It is believed that with in-depth research, the advancement of technology and the deep integration of biomedicine and clinical medicine, patients with arthritis will be offered safer and more effective therapies in the immediate future, and this knowledge will also help us to develop novel treatment strategies.

\section{Abbreviations}

OA: Osteoarthritis; ERRs: Estrogen-related receptors; ERRa/Esrra: Estrogenrelated receptor a; ERRß/Esrrb: Estrogen-related receptor $\beta$; ERRy/Esrrg: Estrogen-related receptor $\gamma ;$ ERa: Estrogen receptor a; ERR: Estrogen receptor $\beta$; DBD: DNA-binding domain; LBD: Ligand-binding domain; AF-1: Activation domain-1; AF-2: Activation function-2; PGC1 a: Peroxisome proliferator-activated receptor $\gamma$ coactivator $1 a$; NCOR1: Nuclear receptor corepressor 1; BAT: Brown adipose tissue; HK2: Hexokinase 2; GAPDH: Glyceraldehyde phosphate dehydrogenase; ENO1: Enolase 1; WAT: White adipose tissue; UCP1: Uncoupling protein 1; ER-: Estrogen receptor-negative; BMMs: Bone marrow-derived macrophages; VEGF: Vascular endothelial growth factor; TNBC: Triple negative 
breast cancer; EMT: Epithelial-mesenchymal transition; TGF: Transforming growth factor; KO: Knockout; Sox-9: Sry-type high-mobility-group box transcription factor 9; IL-1 $\beta$ : Interleukin-1 $\beta$; MMP-13: Matrix metalloproteinase-13; ECM: Extracellular matrix; ROS: Reactive oxygen species; IL-6: Interleukin-6; MMP-9: Matrix metalloproteinase-9; VEGFA: Vascular endothelial growth factor A1; DMM: Destabilization of the medial meniscus; MMP-3: Matrix metalloproteinase-3; p27: Cyclin-dependent kinase inhibitor 1B; DES: Diethylstillbestrol; 4-OHT: 4-Hydroxytamoxifen; TAM: Tamoxifen.

\section{Acknowledgements}

Not applicable.

\section{Authors' contributions}

TJ and ZJ conceived the article. TJ is the major contributor in writing the manuscript and the figures. $L T$ and $Z Z$ created the figures and edited the text. WX, GJ and YJ performed literature search, and provided valuable comments. All authors read and approved the final manuscript.

\section{Funding}

The study was financially supported by the National Natural Science Foundation of China (No. 81601908) and the Science and Technology Development Program of Jilin Province (Nos. 20180520108JH, 20190304124YY).

\section{Availability of data and materials}

All data are available through cited literature.

\section{Ethics approval and consent to participate}

Not applicable.

\section{Consent for publication}

Not applicable.

\section{Competing interests}

The authors declared that they have no competing interests.

\section{Author details}

${ }^{1}$ Department of Orthopeadics, China-Japan Union Hospital of Jilin University, Changchun 130033, Jilin, China. ${ }^{2}$ Department of Hand Surgery, China-Japan Union Hospital of Jilin University, Changchun 130033, Jilin, China.

Received: 5 August 2020 Accepted: 8 January 2021

Published online: 15 January 2021

\section{References}

Aho OM, Finnila M, Thevenot J, Saarakkala S, Lehenkari P. Subchondral bone histology and grading in osteoarthritis. PLoS ONE. 2017;12:e0173726.

Audet-Walsh E, Giguere V. The multiple universes of estrogen-related receptor alpha and gamma in metabolic control and related diseases. Acta Pharmacol Sin. 2015;36:51-61.

Bae $\mathrm{S}$, et al. MYC-dependent oxidative metabolism regulates osteoclastogenesis via nuclear receptor ERRalpha. J Clin Invest. 2017;127:2555-68.

Berman AY, et al. ERRalpha regulates the growth of triple-negative breast cancer cells via S6K1-dependent mechanism. Signal Transduct Target Ther. 2017;2:1-9.

Bielli P, Pagliarini V, Pieraccioli M, Caggiano C, Sette C. Splicing dysregulation as oncogenic driver and passenger factor in brain tumors. Cells. 2019;9:10.

Boehme KA, Rolauffs B. Onset and progression of human osteoarthritis-can growth factors, inflammatory cytokines, or differential miRNA expression concomitantly induce proliferation, ECM degradation, and inflammation in articular cartilage? Int J Mol Sci. 2018;19:2282.

Bombail V, Collins F, Brown P, Saunders PT. Modulation of ER alpha transcriptional activity by the orphan nuclear receptor ERR beta and evidence for differential effects of long- and short-form splice variants. Mol Cell Endocrinol. 2010;314:53-61.

Bonnelye E, Aubin JE. Estrogen receptor-related receptor alpha: a mediator of estrogen response in bone. J Clin Endocrinol Metab. 2005;90:3115-21.

Bonnelye $E$, Aubin JE. An energetic orphan in an endocrine tissue: a revised perspective of the function of estrogen receptor-related receptor alpha in bone and cartilage. J Bone Miner Res. 2013;28:225-33.
Bonnelye $\mathrm{E}$, et al. The ERR-1 orphan receptor is a transcriptional activator expressed during bone development. Mol Endocrinol. 1997;11:905-16.

Bonnelye E, Zirngibl RA, Jurdic P, Aubin JE. The orphan nuclear estrogen receptor-related receptor-alpha regulates cartilage formation in vitro: implication of Sox9. Endocrinology. 2007;148:1 195-205.

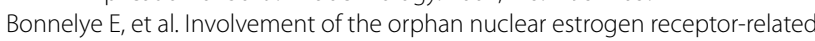
receptor alpha in osteoclast adhesion and transmigration. J Mol Endocrinol. 2010;45:365-77.

Bonnelye E, Reboul P, Duval N, Cardelli M, Aubin JE. Estrogen receptor-related receptor a regulation by interleukin-1 $\beta$ in prostaglandin $E(2)$ - and CAMP-dependent pathways in osteoarthritic chondrocytes. Arthritis Rheum. 2011;63:2374-84.

Brown EL, et al. Estrogen-related receptors mediate the adaptive response of brown adipose tissue to adrenergic stimulation. iscience. 2018a;2:221-37.

Brown EL, et al. PGC-1alpha and PGC-1 beta increase protein synthesis via ERRalpha in C2C12 myotubes. Front Physiol. 2018b;9:1336.

Cardelli M, Aubin JE. ERRgamma is not required for skeletal development but is a RUNX2-dependent negative regulator of postnatal bone formation in male mice. PLoS ONE. 2014;9:e109592.

Cardelli M, et al. Cartilage-specific overexpression of ERRgamma results in Chondrodysplasia and reduced chondrocyte proliferation. PLOS ONE. 2013;8:e81511.

Carnesecchi J, Vanacker JM. Estrogen-related receptors and the control of bone cell fate. Mol Cell Endocrinol. 2016;432:37-43.

Casaburi I, et al. Cholesterol as an endogenous ERRalpha agonist: a new perspective to cancer treatment. Front Endocrinol. 2018;9:525.

Chan CM, et al. Cytokine-induced MMP13 expression in human chondrocytes is dependent on activating transcription factor 3 (ATF3) regulation. J Biol Chem. 2017;292:1625-36.

Charlier E, et al. Chondrocyte dedifferentiation and osteoarthritis (OA). Biochem Pharmacol. 2019;165:49-65.

Chen S, Zhou D, Yang C, Sherman M. Molecular basis for the constitutive activity of estrogen-related receptor alpha-1.J Biol Chem. 2001;276:28465-70.

Chen $\mathrm{X}$, et al. Impact of estrogen-related receptor a on the biological characteristics of rat mandibular condylar chondrocytes. Mol Med Rep. 2014;10:195-202.

Correa D, Lietman SA. Articular cartilage repair: current needs, methods and research directions. Semin Cell Dev Biol. 2017;62:67-77.

Coward P, Lee D, Hull MV, Lehmann JM. 4-Hydroxytamoxifen binds to and deactivates the estrogen-related receptor gamma. Proc Natl Acad Sci USA. 2001;98:8880-4.

Dadabo J, Fram J, Jayabalan P. Noninterventional therapies for the management of knee osteoarthritis. J Knee Surg. 2019;32:46-54.

De Vitto H, Bode AM, Dong Z. The PGC-1/ERR network and its role in precision oncology. NPJ Precis Oncol. 2019;3:9.

Delhon I, et al. Absence of estrogen receptor-related-alpha increases osteoblastic differentiation and cancellous bone mineral density. Endocrinology. 2009;150:4463-72.

DeRogatis $\mathrm{M}$, et al. Non-operative treatment options for knee osteoarthritis. Ann Transl Med. 2019;7:S245.

Divekar SD, Tiek DM, Fernandez A, Riggins RB. Estrogen-related receptor beta (ERRbeta) -renaissance receptor or receptor renaissance? Nucl Recept Signal. 2016;14:e002.

Eichner LJ, Giguere V. Estrogen related receptors (ERRs): a new dawn in transcriptional control of mitochondrial gene networks. Mitochondrion. 2011;11:544-52.

Eudy JD, et al. Isolation of a gene encoding a novel member of the nuclear receptor superfamily from the critical region of Usher syndrome type lla at 1q41. Genomics. 1998;50:382-4.

Fan W, Evans R. PPARs and ERRs: molecular mediators of mitochondrial metabolism. Curr Opin Cell Biol. 2015;33:49-54.

Fang $\mathrm{H}$, et al. Early changes of articular cartilage and subchondral bone in the DMM mouse model of osteoarthritis. Sci Rep. 2018;8:2855.

Festuccia N, Owens N, Navarro P. Esrrb, an estrogen-related receptor involved in early development, pluripotency, and reprogramming. FEBS Lett. 2018;592:852-77.

Fosang AJ, Last K, Knäuper V, Murphy G, Neame PJ. Degradation of cartilage aggrecan by collagenase-3 (MMP-13). FEBS Lett. 1996;380:17-20. 
Fradet $A$, et al. Dual function of ERRa in breast cancer and bone metastasis formation: implication of VEGF and osteoprotegerin. Cancer Res. 2011:71:5728-38.

Gademan MG, Hofstede SN, Vliet Vlieland TP, Nelissen RG, Marang-van de Mheen PJ. Indication criteria for total hip or knee arthroplasty in osteoarthritis: a state-of-the-science overview. BMC Musculoskelet Disord. 2016;17:463.

Gallet M, Vanacker JM. ERR receptors as potential targets in osteoporosis. Trends Endocrinol Metab. 2010;21:637-41.

Gantner ML, Hazen BC, Eury E, Brown EL, Kralli A. Complementary roles of estrogen-related receptors in brown adipocyte thermogenic function. Endocrinology. 2016;157:4770-81.

Gibson DA, Saunders PT. Estrogen dependent signaling in reproductive tissues - a role for estrogen receptors and estrogen related receptors. Mol Cell Endocrinol. 2012;348:361-72.

Giguère V. To ERR in the estrogen pathway. Trends Endocrinol Metab. 2002;13:220-5.

Giquère $V$, Yang N, Segui P, Evans RM. Identification of a new class of steroid hormone receptors. Nature. 1988;331:91-4.

Glyn-Jones S, et al. Osteoarthritis. Lancet. 2015;386:376-87.

Greschik H, et al. Structural and functional evidence for ligand-independent transcriptional activation by the estrogen-related receptor 3. Mol Cell. 2002;9:303-13.

Guan M, et al. Low-intensity pulsed ultrasound inhibits VEGFA expression in chondrocytes and protects against cartilage degeneration in experimental osteoarthritis. FEBS Open Bio. 2020;10:434-43.

Guo W, et al. MMP-3 gene polymorphisms are associated with increased risk of osteoarthritis in Chinese men. Oncotarget. 2017;8:79491-7.

Hamilton JL, et al. Targeting VEGF and Its receptors for the treatment of osteoarthritis and associated pain. J Bone Miner Res. 2016;31:911-24.

Hardy E, Fernandez-Patron C. Destroy to rebuild: the connection between bone tissue remodeling and matrix metalloproteinases. Front Physiol. 2020;11:47.

Harrell CR, Markovic BS, Fellabaum C, Arsenijevic A, Volarevic V. Mesenchymal stem cell-based therapy of osteoarthritis: current knowledge and future perspectives. Biomed Pharmacother. 2019;109:2318-26.

Hashimoto S, et al. Development and regulation of osteophyte formation during experimental osteoarthritis. Osteoarthr Cartil. 2002;10:180-7.

Heckler MM, Riggins RB. ERRbeta splice variants differentially regulate cell cycle progression. Cell Cycle. 2015;14:31-45.

Heckler MM, et al. Antimitotic activity of DY131 and the estrogen-related receptor beta 2 (ERRß2) splice variant in breast cancer. Oncotarget. 2016;7:47201-20.

Helsen C, Claessens F. Looking at nuclear receptors from a new angle. Mol Cell Endocrinol. 2014;382:97-106.

Hirota $\mathrm{K}$, et al. Live imaging analysis of the growth plate in a murine long bone explanted culture system. Sci Rep. 2018;8:10332.

Hong JI, Park IY, Kim HA. Understanding the molecular mechanisms underlying the pathogenesis of arthritis pain using animal models. Int J Mol Sci. 2020;21:533.

Horard B, et al. Dimerization is required for transactivation by estrogen-receptor-related (ERR) orphan receptors: evidence from amphioxus ERR. J Mol Endocrinol. 2004;33:493-509.

Huang T, et al. Aging reduces an ERRalpha-directed mitochondrial glutaminase expression suppressing glutamine anaplerosis and osteogenic differentiation of mesenchymal stem cells. Stem Cells. 2017;35:411-24.

Huo D, Anderson D, Palmer JR, Herbst AL. Incidence rates and risks of diethylstilbestrol-related clear-cell adenocarcinoma of the vagina and cervix: update after 40-year follow-up. Gynecol Oncol. 2017;146:566-71.

Huss JM, Garbacz WG, Xie W. Constitutive activities of estrogen-related receptors: transcriptional regulation of metabolism by the ERR pathways in health and disease. Biochim Biophys Acta. 2015;1852:1912-27.

Jeong BC, et al. The orphan nuclear receptor estrogen receptor-related receptor gamma negatively regulates BMP2-induced osteoblast differentiation and bone formation. J Biol Chem. 2009;284:14211-8.

Johnson VL, Hunter DJ. The epidemiology of osteoarthritis. Best Pract Res Clin Rheumatol. 2014;28:5-15.
Joo J, et al. In vitro metabolism of an estrogen-related receptor $y$ modulator, GSK5182, by human liver microsomes and recombinant cytochrome P450s. Biopharm Drug Dispos. 2015:36:163-73.

Kang $\mathrm{MH}$, et al. Estrogen-related receptor gamma functions as a tumor suppressor in gastric cancer. Nat Commun. 2018;9:1920.

Kashiwagi A, Schipani E, Fein MJ, Greer PA, Shimada M. Targeted deletion of Capn4 in cells of the chondrocyte lineage impairs chondrocyte proliferation and differentiation. Mol Cell Biol. 2010;30:2799-810.

Kayakabe K, et al. Interleukin- 6 promotes destabilized angiogenesis by modulating angiopoietin expression in rheumatoid arthritis. Rheumatology. 2012;51:1571-9.

Kida YS, et al. ERRs mediate a metabolic switch required for somatic cell reprogramming to pluripotency. Cell Stem Cell. 2015;16:547-55.

Kim Y, Koh M, Kim DK, Choi HS, Park SB. Efficient discovery of selective small molecule agonists of estrogen-related receptor gamma using combinatorial approach. J Comb Chem. 2009;11:928-37.

Kim DK, et al. Inverse agonist of nuclear receptor ERRgamma mediates antidiabetic effect through inhibition of hepatic gluconeogenesis. Diabetes. 2013;62:3093-102.

Kim Yl, et al. Cartilage development requires the function of Estrogen-related receptor alpha that directly regulates sox 9 expression in zebrafish. Sci Rep. 2015:5:18011.

Kim J, et al. Identification of selective ERRgamma inverse agonists. Molecules. 2016;21:80

Kim HJ, et al. Estrogen-related receptor $\gamma$ negatively regulates osteoclastogenesis and protects against inflammatory bone loss. J Cell Physiol. 2019a:234:1659-70.

Kim J, et al. Discovery of potent, selective, and orally bioavailable estrogenrelated receptor- $\gamma$ inverse agonists to restore the sodium iodide symporter function in anaplastic thyroid cancer. J Med Chem. 2019b:62:1837-58.

Kokabu T, et al. Antitumor effect of XCT790, an ERRalpha inverse agonist, on ERalpha-negative endometrial cancer cells. Cell Oncol. 2019:42:223-35.

Kotula-Balak M, et al. Insights into the role of estrogen-related receptors alpha, beta and gamma in tumor Leydig cells. Tissue Cell. 2018;52:78-91.

Kulshrestha V, Datta B, Mittal G, Kumar S. Epidemiology of revision total knee arthroplasty: a single center's experience. Indian J Orthop. 2019:53:282-8.

Leopold Wager CM, Arnett E, Schlesinger LS. Macrophage nuclear receptors: emerging key players in infectious diseases. PLoS Pathog. 2019;15:e1007585.

Li H, Wang D, Yuan Y, Min J. New insights on the MMP-13 regulatory network in the pathogenesis of early osteoarthritis. Arthritis Res Ther. 2017;19:248.

Li $P$, et al. ERRalpha is an aggressive factor in lung adenocarcinoma indicating poor prognostic outcomes. Cancer Manag Res. 2019a;11:8111-23.

$\mathrm{Li}$ D, et al. Computational insights into the interaction mechanisms of estrogen-related receptor alpha with endogenous ligand cholesterol. Chem Biol Drug Des. 2019b;94:1316-29.

Li X, Zhang K, Hu Y, Luo N. ERRalpha activates SHMT2 transcription to enhance the resistance of breast cancer to lapatinib via modulating the mitochondrial metabolic adaption. Biosci Rep. 2020. https://doi. org/10.1042/BSR20192465.

Liao CR, et al. Advanced oxidation protein products increase TNF-alpha and IL-1 beta expression in chondrocytes via NADPH oxidase 4 and accelerate cartilage degeneration in osteoarthritis progression. Redox Biol. 2020;28:101306.

Likhite $\mathrm{N}$, et al. Loss of estrogen-related receptor alpha facilitates angiogenesis in endothelial cells. Mol Cell Biol. 2019;39:e00411-18.

Lima TI, et al. Role of NCoR1 in mitochondrial function and energy metabolism. Cell Biol Int. 2018:42:734-41.

Liu X, et al. a-Helix-peptides comprising the human nuclear receptor ERRY competitively provoke inhibition of functional homomeric dimerization. Biopolymers. 2016;106:547-54.

Liu CF, Angelozzi M, Haseeb A, Lefebvre V. SOX9 is dispensable for the initiation of epigenetic remodeling and the activation of marker genes at the onset of chondrogenesis. Development. 2018. https://doi.org/10.1242/ dev. 164459 .

Loeser RF, Goldring SR, Scanzello CR, Goldring MB. Osteoarthritis: a disease of the joint as an organ. Arthritis Rheum. 2012;64:1697-707. 
Long W, et al. Estrogen-related receptor participates in regulating glycolysis and influences embryonic development in silkworm Bombyx mori. Insect Mol Biol. 2020;29:160-9.

Lorenzo J. The many ways of osteoclast activation. J Clin Invest. 2017;127:2530-2.

Lu X, Peng L, Lv M, Ding K. Recent advance in the design of small molecular modulators of estrogen-related receptors. Curr Pharm Des. 2012;18:3421-31.

Lu Z, et al. Identification, characterization and mRNA transcript abundance profiles of estrogen related receptor (ERR) in Sepiella japonica imply its possible involvement in female reproduction. Anim Reprod Sci. 2019;211:106231.

Manna S, et al. ERRalpha is a marker of tamoxifen response and survival in triple-negative breast cancer. Clin Cancer Res. 2016;22:1421-31.

Mathiessen A, Conaghan PG. Synovitis in osteoarthritis: current understanding with therapeutic implications. Arthritis Res Ther. 2017;19:18

McCulloch K, Litherland GJ, Rai TS. Cellular senescence in osteoarthritis pathology. Aging Cell. 2017;16:210-8.

Mehana EE, Khafaga AF, El-Blehi SS. The role of matrix metalloproteinases in osteoarthritis pathogenesis: an updated review. Life Sci. 2019;234:116786.

Michigami T. Regulatory mechanisms for the development of growth plate cartilage. Cell Mol Life Sci. 2013;70:4213-21.

Millerand M, Berenbaum F, Jacques C. Danger signals and inflammaging in osteoarthritis. Clin Exp Rheumatol. 2019;37(Suppl 120):48-56.

Misawa A, Inoue S. Estrogen-related receptors in breast cancer and prostate cancer. Front Endocrinol. 2015:6:83.

Misra J, et al. O-GlcNAcylation of orphan nuclear receptor estrogenrelated receptor $y$ promotes hepatic gluconeogenesis. Diabetes. 2016;65:2835-48.

Misra J, Kim DK, Choi HS. ERRy: a junior orphan with a senior role in metabolism. Trends Endocrinol Metab. 2017;28:261-72.

Mohideen-Abdul K, et al. Importance of the sequence-directed DNA shape for specific binding site recognition by the estrogen-related receptor. Front Endocrinol. 2017:8:140.

Murphy NJ, Eyles JP, Hunter DJ. Hip osteoarthritis: etiopathogenesis and implications for management. Adv Ther. 2016;33:1921-46.

Ning Y, et al. A novel compound Ling H2-10 inhibits the growth of triple negative breast cancer cells in vitro and in vivo as a selective inverse agonist of estrogen-related receptor alpha. Biomed Pharmacother. 2017;93:913-22.

Oelkrug R, Polymeropoulos ET, Jastroch M. Brown adipose tissue: physiological function and evolutionary significance. J Comp Physiol B. 2015;185:587-606.

Park S, et al. ERRalpha-regulated lactate metabolism contributes to resistance to targeted therapies in breast cancer. Cell Rep. 2016;15:323-35.

Patch RJ, et al. Identification of diaryl ether-based ligands for estrogenrelated receptor a as potential antidiabetic agents. J Med Chem. 2011:54:788-808.

Porter C. Quantification of UCP1 function in human brown adipose tissue. Adipocyte. 2017;6:167-74

Rahmati M, Nalesso G, Mobasheri A, Mozafari M. Aging and osteoarthritis: central role of the extracellular matrix. Ageing Res Rev. 2017;40:20-30.

Saito K, Cui H. Emerging roles of estrogen-related receptors in the brain: potential interactions with estrogen signaling. Int J Mol Sci. 2018;19:1091.

Schwartz AM, Farley KX, Guild GN, Bradbury TL Jr. Projections and epidemiology of revision hip and knee arthroplasty in the United States to 2030. J Arthroplasty. 2020;35:S79-85.

Semerano $L$, et al. Targeting VEGF-A with a vaccine decreases inflammation and joint destruction in experimental arthritis. Angiogenesis. 2016;19:39-52.

Smith EK, White MC, Weir HK, Peipins LA, Thompson TD. Higher incidence of clear cell adenocarcinoma of the cervix and vagina among women born between 1947 and 1971 in the United States. Cancer Causes Control. 2012;23:207-11.

Son YO, Chun JS. Estrogen-related receptor gamma is a novel catabolic regulator of osteoarthritis pathogenesis. BMB Rep. 2018;51:165-6.

Son YO, et al. Estrogen-related receptor gamma causes osteoarthritis by upregulating extracellular matrix-degrading enzymes. Nat Commun. 2017:8:2133.
Sonoda J, et al. Nuclear receptor ERR alpha and coactivator PGC-1 beta are effectors of IFN-gamma-induced host defense. Genes Dev. 2007:21:1909-20.

Suetsugi M, Su L, Karlsberg K, Yuan YC, Chen S. Flavone and isoflavone phytoestrogens are agonists of estrogen-related receptors. Mol Cancer Res. 2003;1:981-91.

Tabeian $\mathrm{H}$, et al. IL-1 beta damages fibrocartilage and upregulates MMP-13 expression in fibrochondrocytes in the condyle of the temporomandibular joint. Int J Mol Sci. 2019;20:2260.

Tetlow LC, Adlam DJ, Woolley DE. Matrix metalloproteinase and proinflammatory cytokine production by chondrocytes of human osteoarthritic cartilage: associations with degenerative changes. Arthritis Rheum. 2001:44:585-94.

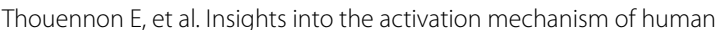
estrogen-related receptor gamma by environmental endocrine disruptors. Cell Mol Life Sci. 2019;76:4769-81.

Tiek DM, et al. Estrogen-related receptor $\beta$ activation and isoform shifting by cdc2-like kinase inhibition restricts migration and intracranial tumor growth in glioblastoma. FASEB J. 2019;33:13476-91.

Titus $L$, et al. Reproductive and hormone-related outcomes in women whose mothers were exposed in utero to diethylstilbestrol (DES): a report from the US National Cancer Institute DES Third Generation Study. Reprod Toxicol. 2019;84:32-8.

Tremblay AM, Wilson BJ, Yang XJ, Giguère V. Phosphorylation-dependent sumoylation regulates estrogen-related receptor-alpha and-gamma transcriptional activity through a synergy control motif. Mol Endocrinol. 2008;22:570-84

Tribollet $\mathrm{V}$, et al. miR-135a inhibits the invasion of cancer cells via suppression of ERRalpha. PLoS ONE. 2016:11:e0156445.

Tripathi M, Yen PM, Singh BK. Estrogen-related receptor alpha: an underappreciated potential target for the treatment of metabolic diseases. Int J Mol Sci. 2020;21:1645.

Vernier M, et al. Estrogen-related receptors are targetable ROS sensors. Genes Dev. 2020;34:544-59.

Villena JA, Kralli A. ERRalpha: a metabolic function for the oldest orphan. Trends Endocrinol Metab. 2008;19:269-76.

Vinatier C, Merceron C, Guicheux J. Osteoarthritis: from pathogenic mechanisms and recent clinical developments to novel prospective therapeutic options. Drug Discov Today. 2016;21:1932-7.

Vu EH, Kraus RJ, Mertz JE. Phosphorylation-dependent sumoylation of estrogen-related receptor alpha1. Biochemistry. 2007;46:9795-804.

Wan Y. PPARy in bone homeostasis. Trends Endocrinol Metab. 2010:21:722-8.

Wang T, He C. Pro-inflammatory cytokines: the link between obesity and osteoarthritis. Cytokine Growth Factor Rev. 2018;44:38-50.

Wang Y, et al. TNF-alpha-induced LRG1 promotes angiogenesis and mesenchymal stem cell migration in the subchondral bone during osteoarthritis. Cell Death Dis. 2017a;8:e2715.

Wang $\mathrm{S}$, et al. Chondrogenic progenitor cells promote vascular endothelial growth factor expression through stromal-derived factor-1. Osteoarthr Cartil. 2017b;25:742-9.

Watt FE. Hand osteoarthritis, menopause and menopausal hormone therapy. Maturitas. 2016:83:13-8.

Wei W, et al. Ligand activation of ERRalpha by cholesterol mediates statin and bisphosphonate effects. Cell Metab. 2016;23:479-91.

Wojdasiewicz P, Poniatowski LA, Szukiewicz D. The role of inflammatory and anti-inflammatory cytokines in the pathogenesis of osteoarthritis. Mediat Inflamm. 2014;2014:561459.

Wu SC, et al. Hyaluronan size alters chondrogenesis of adipose-derived stem cells via the CD44/ERK/SOX-9 pathway. Acta Biomater. 2018;66:224-37.

Xia $H$, Dufour $C R$, Giguère V. ERRa as a bridge between transcription and function: role in liver metabolism and disease. Front Endocrinol. 2019;10:206.

$\mathrm{Xu} Z$, et al. Research progress on the reproductive and non-reproductive endocrine tumors by estrogen-related receptors. J Steroid Biochem Mol Biol. 2016;158:22-30.

Yang D, Wan Y. Molecular determinants for the polarization of macrophage and osteoclast. Semin Immunopathol. 2019;41:551-63.

Ye $X$, et al. The enhanced expression of estrogen-related receptor a in human bladder cancer tissues and the effects of estrogen-related receptor a knockdown on bladder cancer cells. J Cell Biochem. 2019:120:13841-52 
Yoriki K, et al. Estrogen-related receptor alpha induces epithelial-mesenchymal transition through cancer-stromal interactions in endometrial cancer. Sci Rep. 2019;9:6697.

Yu DD, Huss JM, Li H, Forman BM. Identification of novel inverse agonists of estrogen-related receptors ERRgamma and ERRbeta. Bioorg Med Chem. 2017:25:1585-99.

Zhang LD, et al. Downregulation of ERRalpha inhibits angiogenesis in human umbilical vein endothelial cells through regulating VEGF production and PI3K/Akt/STAT3 signaling pathway. Eur J Pharmacol. 2015;769:167-76.

Zhang L, Wong J, Vanacker JM. The estrogen-related receptors (ERRs): potential targets against bone loss. Cell Mol Life Sci. 2016;73:3781-7.

Zhang JF, et al. Expression of matrix metalloproteinases, tissue inhibitors of metalloproteinases, and interleukins in vertebral cartilage endplate. Orthop Surg. 2018a;10:306-11.

Zhang Y, et al. Inverse agonist of ERRY reduces cannabinoid receptor type 1-mediated induction of fibrinogen synthesis in mice with a high-fat diet-intoxicated liver. Arch Toxicol. 2018b;92:2885-96.
Zhang H, Cai D, Bai X. Macrophages regulate the progression of osteoarthritis. Osteoarthr Cartil. 2020;28:555-61.

Zhao $\mathrm{H}$, et al. Estrogen-related receptor gamma induces angiogenesis and extracellular matrix degradation of temporomandibular joint osteoarthritis in rats. Front Pharmacol. 2019;10:1290.

Zhu S, et al. Subchondral bone osteoclasts induce sensory innervation and osteoarthritis pain. J Clin Invest. 2019:129:1076-93.

Zuercher WJ, et al. Identification and structure-activity relationship of phenolic acyl hydrazones as selective agonists for the estrogen-related orphan nuclear receptors ERRbeta and ERRgamma. J Med Chem. 2005;48:3107-9.

\section{Publisher's Note}

Springer Nature remains neutral with regard to jurisdictional claims in published maps and institutional affiliations.
Ready to submit your research? Choose BMC and benefit from:

- fast, convenient online submission

- thorough peer review by experienced researchers in your field

- rapid publication on acceptance

- support for research data, including large and complex data types

- gold Open Access which fosters wider collaboration and increased citations

- maximum visibility for your research: over $100 \mathrm{M}$ website views per year

At $\mathrm{BMC}$, research is always in progress.

Learn more biomedcentral.com/submissions 